\title{
Global Myocardial Work in Coronary Artery Disease Patients Without Regional Wall Motion Abnormality: Correlation With Gensini Score
}

\section{Miao Li}

Nanjing First Hospital https://orcid.org/0000-0002-4360-1960

\section{Yuhao Wang}

Nanjing First Hospital

Lin Li

Nanjing First Hospital

Wenfang Wu

Nanjing First Hospital

Pingyang Zhang ( $\nabla$ zhpy28@126.com )

Nanjing First Hospital

\section{Research Article}

Keywords: Global Myocardial, Abnormality, Gensini Score

Posted Date: November 1st, 2021

DOI: https://doi.org/10.21203/rs.3.rs-908574/v1

License: (c) (i) This work is licensed under a Creative Commons Attribution 4.0 International License. Read Full License 


\section{Abstract}

\section{Purpose}

This study aimed to investigate global myocardial work (GMW), derived from non-invasive left ventricular (LV) pressure-strain loops (PSLs) in coronary artery disease (CAD) patients without regional wall motion abnormality (RWMA), and explored the relationship between GMW and severity of CAD using Gensini score (GS) .

Methods

120 patients prepared for coronary angiography (CAG) who had left ventricular ejection fraction $\geq 55 \%$, no resting RWMA in two-dimensional echocardiography were enrolled. Global longitudinal strain (GLS), GMW parameters (including global myocardial work index (GWI), global constructive work (GCW), global wasted work (GWW) and global myocardial work efficiency (GWE)) were quantified. The severity of coronary lesions was evaluated by GS system based on CAG findings. We divided CAG-confirmed CAD patients into three subgroups according to the tertiles of GS: low $0<\mathrm{GS} 16$, mid $16<\mathrm{GS} 38$, and high GS>38.

Results

CAD patients showed a significantly reduced GLS and GWE, but an increased GWW. GLS, GWE, GWI and GCW were significantly decreased in the high-GS group while GWW was increased. GLS, GWE, GWI and GCW was negatively correlated with the GS, GWW was positively correlated with GS. Multivariate regression analysis showed that GWE was the independent factor of predicting coronary stenosis. ROC analysis demonstrated that GWE was the most powerful predictor of high-GS and was superior to GLS. GWE under $91 \%$ had the optimal sensitivity and specificity for identifying high-GS.

Conclusion

The proposed GWE, which outperformed the GLS, showed the optimal performance and could be considered as a potential predictive indicator to detect severe coronary disease in non-RWMA CAD patients.

\section{Introduction}

Coronary atherosclerotic diseases (CAD) remain the leading cause of mortality and morbidity in patients with cardiovascular diseases [1]. Early detection of coronary artery lesions has a paramount impact on clinical decision and prognosis improvement. Currently, coronary angiography (CAG) has been a standard tool, which was used in CAD diagnosis and estimated the severity of coronary lesion. Gensini score (GS) based on CAG findings has become an effective parameter in predicting survival outcome and adverse cardiovascular events [2, 3]. However, as an invasive examination method, CAG has certain risks, especially for patients with poor general conditions. It is of great importance to seek a non-invasive method to detect CAD and evaluate the degree of coronary artery stenosis. 
Transthoracic echocardiography (TTE) was a wide-accepted non-invasive technique in diagnosing CAD based on detecting decreased left ventricular ejection fraction (LVEF) and regional wall motion abnormality (RWMA). However, during the early stage of coronary atherosclerosis, unless there has been an evident ischemia or infarction, RWMAs are usually invisible and the LV functional parameters tend to be preserved. More recently, speckle-tracking echocardiography (STE) focusing on myocardial strain analysis has been gaining ground. Previous studies have shown that myocardial strain parameters significantly decreased in CAD patients, even when LVEF and resting LV wall motion are normal. Although STE provides a new perspective to quantify myocardial function beyond conventional assessment of RWMAs[4-7], load dependence is the main factor to affect the diagnostic accuracy of STE in the evaluation of myocardial function [8].

Myocardial work (MW) analysis is a novel method accounts for myocardial strain as well as afterload offering comprehensive quantitative assessment of myocardial function $[9,10]$. Chan et al applied MW analysis in patients with hypertension (HTN) [11]. The result showed that global work index (GWI) in patients with HTN was significantly increased compared to healthy controls while GLS and LVEF remained constant. This finding verified the apparent limitation of conventional methods in detecting myocardial performance in patients with increased afterload. Recently, Boe E et al. revealed that in patients with non-ST-segment elevation acute coronary syndrome, regional MW analysis can be used to identify the presence of acute coronary occlusion [8].

, The aim of this study was to explore the feasibility of MW analysis in CAD detection, and identify the correlation between MW and coronary lesion severity determined from the Gensini score.

\section{Methods}

\subsection{Clinical enrollment}

All 120 patients diagnosed with CAD referred for CAG from July 2020 to January 2021 were retrieved from Nanjing First Hospital. TTE was applied on patients within 12 hours before CAG. 41 patients were excluded because of evident RWMA on TTE; LVEF $<55 \%$; significant valvular heart diseases; left ventricular outflow tract (LVOT) obstruction; poor echocardiographic image quality and severe systemic diseases (Figure 1).

Institutional review board of Nanjing First Hospital approved this study, and informed consent was obtained from all individual participants in current study.

\section{Routine TTE}

All conventional two-dimensional echocardiographic parameters were performed in accordance with the guidelines of the American Society of Echocardiography. All participants underwent electrocardiograph (ECG) monitoring during TTE examination. TTE was performed $<12$ hours before CAG using a Vivid E95 ultrasound system (GE Healthcare, Horten, Norway) equipped with an M5S transducer (3.5MHz). Stroke 
volume (SV) and LVEF (\%) were calculated using the modified bi-plane Simpson method. peak E/A ratio and peak $\mathrm{E} / \mathrm{e}$ ratio of the mitral valve (MV) were obtained by spectral doppler and tissue doppler imaging.

\section{Two-Dimensional STE}

Apical long-axis, and apical two and four chamber view standard two-dimensional images consisting of 3 cardiac circles were acquired for offline analysis. For the successful implementation of STE, the frame rates were set $\geq 80$ frames/sec. Global longitudinal strain (GLS) was quantified by sonographers who are unaware of CAG results using semi-automated functional imaging using EchoPAC software (version 202, GE Vingmed Ultrasound). Automatic algorithm traced and tracked LV myocardium. The endocardium boundary can be adjusted manually if necessary. Weighted average value of segmental peak longitudinal strains was used to calculate GLS.

\section{MW Analysis}

MW parameters were calculated using EchoPAC software (version 202, GE Vingmed Ultrasound). The methodology of MW measurement derived from non-invasive pressure-strain loops (PSLs) has been described previously[9] . The peak systolic LV pressure was estimated by peak arterial pressure using sphygmomanometer during TTE. The adjusted non-invasive LV pressure curve was demonstrated by MW software based on duration of isovolumic and ejection phases. On the basis of GLS data obtained by STE mentioned above, MW was then quantified by calculating the rate of segmental shortening by differentiation of the strain curve and multiplying this value with instantaneous LV pressure. Following indices were calculated automatically by software including: global myocardial work index (GWI), total work within the area of the LV PSL calculated from mitral valve closure to mitral valve opening; global constructive work(GCW), global myocardial work performed during LV shortening in systole and LV lengthening during the isovolumic relaxation phase; global myocardial wasted work(GWW), global myocardial work performed during LV lengthening in systole and LV shortening during the isovolumic relaxation phase; global myocardial work efficiency(GWE), the ratio between GCW and the sum of GCW and GWW.

\section{CAG examination and Gensini scoring system}

Two certified senior cardiologists independently assessed all angiograms, both were blinded of patient's clinicopathological information. Gensini scoring system was used to evaluate the severity of coronary artery stenosis [2] (Supplementary Table 1). In present study, the tertile stratification was used to divided CAG-confirmed CAD patients into the low-GS, middle-GS, and high-GS groups with Gensini scores of $£ 16$, 17-38, $>38$, respectively.

\section{Variability analysis}

The clinical data and variability analysis were manually delineated from 10 randomly selected patients by the prime observer and an experienced cardiac sonographer in double-blind method. The prime observer 
reported repeated GLS and MW parameters measurements one week after original measurements. Intraand inter-observer variabilities were calculated by intra-class correlation coefficient (ICC).

\section{Statistical analysis}

All statistical analyses were conducted on SPSS version 21.0 (Chicago, IL, USA) and R software 3.6.2 (Vienna, Austria). Data are presented as mean \pm standard deviation for normally distributed continuous variables, and as numbers and percentages for categorical variables. The independent student $T$ test was used for inter-group comparison, univariate analysis was used for continuous variables, and $\mathrm{c}^{2}$-test was used for categorical variables. Pearson correlation coefficient was applied to evaluate the correlation between clinical variables. Multiple linear regression analysis was performed to determine the factors affecting GS. A receiver operating characteristic (ROC) analysis was used to identify the best cutoff values of GLS and MW parameters able to predict high-GS. A two tailed $p$-value $<0.05$ was considered statistically significant.

\section{Results}

\section{Patient characteristics}

A total of 79 individuals were eligible for this study. Based on the CAG findings, we divided all subjects (mean age, 61.80 \pm 10.75 years; 71 male) into two groups, CAD patients $(n=62)$ and no CAD (defined as control group, $n=17$ ). Patient baseline clinical and echocardiographic variables are presented in Table 1 . Patients in CAD group were significantly elder than control control $(63.32 \pm 10.38$ vs $56.24 \pm 10.86$ years $\mathrm{p}=0.016)$.

Smokers were more common in CAD group. Also, higher systolic blood pressure (SBP)was found in CAD patients compared to control. Remarkably, LVEF between CAD and control showed no significantly difference $(p=0.524)$.

All 62 CAD patients were then further sub-divided into three groups (21 patients in low-GS group (33.8\%), 22 patients in mid-GS group (35.4\%), 19 patients in high-GS group (30.6\%)) according to the tertiles of GS.

All patients enrolled in this study remained stable condition during the imaging examinations.

\section{GLS and GMW parameters in CAD group and control subjects}

GLS and GMW variables in all participants were summarized in Table 2. Statistical analyses revealed that GLS and GWE in CAD group were significantly reduced when compared to control subjects. In contrast, GWW was increased in CAD patients $(p<0.05)$. There is no significant difference in GWI and GCW between $C A D$ group and the control group ( $p>0.05$ ). Figure 2 represented a typical diagram of GMW parameters from one patient with no angiographic evidence of $C A D$ and one patient with severe $C A D(G S=42)$. 


\section{Clinicopathological and echocardiographic features, GLS \& GMW variables in low-, mid-, and high- GS groups.}

Baseline clinicopathological characteristics and conventional echocardiographic variables of patients involved in this study are shown in Supplementary Table 2. In high-GS group, age and IVSd were significantly higher than that in low- and mid-GS groups $(p<0.05)$. Apart from this, hyperlipidemia was more common among patients with high-GS. $(p=0.019)$.

GLS was the conventional parameter to evaluate the myocardial strain, our results showed that GLS was significantly decreased in high-GS group (Fig.3a). Using myocardial work analysis, we noticed that GWE, GWI and GCW were obviously lower in high-GS group than that in the other two groups, which objectively revealed the relationship between LV myocardial work performance and the severity of coronary artery stenosis (Fig.3b, c\& d). Consistent with our findings, GWW, served as the parameter demonstrated the global myocardial wasted work, was increased in high-GS group (Fig.3e).

\section{Correlation ship between GLS, GMW parameters and GS}

In Table 3, our results revealed that GLS, GWE, GWI, GCW and GS were negatively interrelated $(r=-0.478$ (GLS vs GS), -0.708 (GWE vs GS), -0.454 (GWI vs GS), -0.449 (GCW vs GS) respectively; Pearson correlation test, all $p<0.05)$, and a positive correlation between GWW and $G S$. $(r=0.505, p<0.05)$. The value of area under ROC curves (AUC) of GLS, GWE, GWI, GCW and GWW were 0.677, 0.839, 0.669, 0.677 and 0.796 respectively, for the identification of severe coronary lesions (high-GS) (Table 4). GWE was superior to GLS and other GMW parameters with an AUC of 0.839. The optimal cutoff value of GWE for assessing non-RWMA in CAD patients with severe coronary lesions (high-GS) was $91 \%$, with sensitivity of $76.19 \%$ and a specificity of $73.17 \%$ (Figure 4). Multivariate regression analysis showed that GWE was the only independent factor of predicting coronary stenosis, which was mainly assessed by GS. ( $\beta=39.330$, $\mathrm{p}=0.001)$ (Table 5).

\section{Inter- and intra-observer variability}

The inter- and intra-observer variability for GLS and GMW parameters were listed in Supplementary Table 3. All parameters exhibited good repeatability, with ICC values $>0.80$.

\section{Discussion}

Currently, the specificity of non-invasive method to diagnose CAD without RWMA is still dismal. Before the emergence of myocardial deformation analysis, stress echocardiography (SE) remains a highly acceptable imaging method for assessing CAD with no RWMA on routine TTE[12]. SE, including exercise stress and pharmacological stress, can enhance the tested object's myocardial contractile to show the ischemic myocardium by increasing cardiac output, which may cause tachycardia, malignant arrhythmia, chest pain potentially. Also, SE has other important limitations, including operator dependence, endocardial border definition, subjectivity, and image quality requirements [12, 13]. 
For the last decade, myocardial deformation analysis based on speckle tracking imaging has become a reliable and comprehensive assessment of myocardial characteristics and systolic left ventricular function. $[14,5]$. Previous studies reported the correlation between myocardial mechanical strain and the severity of coronary artery stenosis in EF-preserved CAD patients[4-7]. However, STE analysis is still limited in being load dependency. The increase of afterload showed negative correlation with strain, which leading to misunderstandings about the real myocardial contractility[9].

\section{Myocardial work analysis}

Myocardial work analysis, derived from non-invasive LV PSL, incorporates LV pressure and strain, therefore show a superior value in assessing LV contractibility to other echocardiographic parameters including LVEF and strain. Previous study demonstrated that regional myocardial glucose metabolism by F-fluorodeoxyglucose positron emission tomography (18F-FDG PET) evaluation showed a strong correlation with myocardial work[9]. The myocardial work methods can estimate the myocardial energetics owing to the PSL area reflects myocardial oxygen consumption and metabolism. Present study demonstrated that non-invasive MW analysis derived by PSLs can detect CAD patients with quantifiable information, even for patients with invisible RWMA. In brief, compared to healthy controls, GLS and GWE in CAD group were significantly reduced, with GWW increased, although LVEF showed no significant difference $(64.63 \pm 1.17$ vs. $65.00 \pm 1.87, p=0.524)$. This result indicated that myocardial deformability and work performance had been impaired in those patients despite the LV ejection function seemed unchanged.

Gensini scoring system, an objective tool to diagnose coronary lesions, mainly estimated the CAD severity depending on the degree of multi-vessel luminal narrowing and anatomical location. Compared to the conventional assessment of coronary stenosis only focuses on the most severe luminal stenosis of coronary artery, Gensini scoring system can assessing CAD severity more comprehensive and valuable information $[15,16]$. In present study, we found that GLS, GWE, GWI and GCW in the high-GS group were reduced while GWW increased compared with low- and mid-GS groups. It has been proven that in a normal heart, different myocardial segments contract in a highly synchronized and coordinated manner[17]. The positive work performed during LV shortening in systole and LV lengthening during the relaxation phase was defined as constructive work. In the meantime, wasted work was defined as the work generated during systolic lengthening and diastolic shortening in dyssynchronous contractions [10]. Therefore, in patients with high-GS, severe myocardial ischemia may induce a conspicuous reduction of myocardial oxygen and blood flow, leading to a decreased myocardial work performance and increased wasted work.

In addition, Our ROC findings demonstrated that GWE was the most powerful predictor of identifying highGS in patients with CAD (AUC value= 0.839) and was superior to GLS (AUC value $=0.677$ ). A GWE value of less than $91 \%$ allowed a sensitivity of $76.19 \%$ and a specificity of $73.17 \%$ for evaluating non-RWMA CAD patients with severe coronary lesions. 'Global myocardial work efficiency' (GWE) is defined as followed: GWE = Constructive work/ (Wasted work + Constructive work), which tend to be a more objective indicator in different patients regardless of individual variation[9]. In present study, multivariate regression analysis 
also elucidated that GWE was the only independent factor of predicting coronary stenosis, which was mainly assessed by GS. $(\beta=39.330, p=0.001)$.

Furthermore, our previous study, which focus on the index change of left ventricular dyssynchrony (LVD) in non-RWMA CAD patients, revealed that CAD patients tended to show increased LVD compared to negative groups[18]. Florian Schrub et al. reported that the myocardial work may play an important role in detecting the presence of LVD in dilated cardiomyopathy patients[7]. Notably, the significant change of GWW and GWE, and a heterogeneous distribution of MW showed observably association with LVD using echocardiography. As a matter of fact, other studies have shown that a delayed onset of segmental subendocardial thinning occurred at an early stage of ischemic hypoperfusion, which can induce regional asynchronous contraction, further leading to presence of $\operatorname{LVD}[19,20]$. These alterations may associate and interact with heterogeneous distribution of myocardial work, leading to an impairment of global myocardial work efficiency and overall myocardial performance[21, 22]. Whether myocardial work and LVD interact with each other or just present as two different symptoms caused by coronary ischemia deserves further investigation.

\section{Limitations}

This study has limitations inherent to its retrospective nature and the study population. Firstly, this study enrolled patients in a single center with a limited number of participants, further external validations based on more large-scale cohorts will seek to test our conclusion. Secondly, instead of invasive left ventricular pressure, brachial artery cuff pressure was used to generate the estimated pressure curve during the assessment of MW by PSLs. We acknowledge that in some situations, when the aortic pressure inconsistent with left ventricular pressure, especially in patients with LVOT obstruction or aortic stenosis, may affect generalizability and predictive accuracy of our study. Therefore, in present study, we enrolled patients without aortic stenosis, LVOT obstruction, and other pressure gradient-related cardiomyopathy. Further investigation is needed to ensure the accuracy and clinical utility of the predictive parameter we demonstrated.

\section{Conclusion}

In summary, LV PSLs can be applied to estimate MW in CAD patients. WM parameters assessment provided incremental value in detecting CAD in non-RWMA patients. The proposed GWE, as a representative parameter of MW, showed the optimal performance in predicting high-GS which could be considered as a potential predictive factor to detect severe coronary artery disease in CAD patients without RWMA.

\section{Declarations}

Acknowledgements We appreciated Yaodong Zhang for his valuable suggestion in statistical analysis.

Funding No Funding has been received by any of the authors. 


\section{Compliance with ethical standards}

Ethics approval All procedures performed in studies involving human participants were in accordance with the ethical standards of the Ethics Committees of Nanjing First Hospital of the Ministry of Health (Nanjing, China) and with the 1964 Helsinki declaration and its later amendments or comparable ethical standards.

Conflict of interest the authors declare that they have no conflict of interest.

Consent to participate Informed consent was obtained from all individual participants included in the study.

\section{References}

1. Lozano R, Naghavi M, Foreman K, Lim S, Shibuya K, Aboyans V et al. Global and regional mortality from 235 causes of death for 20 age groups in 1990 and 2010: a systematic analysis for the Global Burden of Disease Study 2010. Lancet. 2012;380(9859):2095-128. doi:10.1016/S0140-6736(12)61728-0.

2. Gensini GG. A more meaningful scoring system for determining the severity of coronary heart disease. The American journal of cardiology. 1983;51(3):606. doi:10.1016/s0002-9149(83)80105-2.

3. Fu Z, Xue H, Dong W, Chen L, Gai L, Liu H et al. Correlation between comprehensive evaluation of coronary artery lesion severity and long-term clinical outcomes in Chinese octogenarians with acute coronary syndrome. Heart, lung \& circulation. 2014;23(12):1125-31. doi:10.1016/j.hlc.2014.04.260.

4. Li L, Zhang PY, Ran H, Dong J, Fang LL, Ding QS. Evaluation of left ventricular myocardial mechanics by three-dimensional speckle tracking echocardiography in the patients with different graded coronary artery stenosis. The international journal of cardiovascular imaging. 2017;33(10):1513-20. doi:10.1007/s10554-017-1147-6.

5. Luis SA, Yamada A, Khandheria BK, Speranza V, Benjamin A, Ischenko M et al. Use of three-dimensional speckle-tracking echocardiography for quantitative assessment of global left ventricular function: a comparative study to three-dimensional echocardiography. Journal of the American Society of Echocardiography : official publication of the American Society of Echocardiography. 2014;27(3):285-91. doi:10.1016/j.echo.2013.11.002.

6. Winter R, Jussila R, Nowak J, Brodin LA. Speckle tracking echocardiography is a sensitive tool for the detection of myocardial ischemia: a pilot study from the catheterization laboratory during percutaneous coronary intervention. Journal of the American Society of Echocardiography : official publication of the American Society of Echocardiography. 2007;20(8):974-81. doi:10.1016/j.echo.2007.01.029.

7. Biering-Sorensen T, Hoffmann S, Mogelvang R, Zeeberg Iversen A, Galatius S, Fritz-Hansen T et al. Myocardial strain analysis by 2-dimensional speckle tracking echocardiography improves diagnostics of 
coronary artery stenosis in stable angina pectoris. Circulation Cardiovascular imaging. 2014;7(1):58-65. doi:10.1161/CIRCIMAGING.113.000989.

8. Boe E, Russell K, Eek C, Eriksen M, Remme EW, Smiseth OA et al. Non-invasive myocardial work index identifies acute coronary occlusion in patients with non-ST-segment elevation-acute coronary syndrome. European heart journal cardiovascular Imaging. 2015;16(11):1247-55. doi:10.1093/ehjci/jev078.

9. Russell K, Eriksen M, Aaberge L, Wilhelmsen N, Skulstad H, Remme EW et al. A novel clinical method for quantification of regional left ventricular pressure-strain loop area: a non-invasive index of myocardial work. European heart journal. 2012;33(6):724-33. doi:10.1093/eurheartj/ehs016.

10. Boe E, Skulstad H, Smiseth OA. Myocardial work by echocardiography: a novel method ready for clinical testing. European heart journal cardiovascular Imaging. 2019;20(1):18-20.

doi:10.1093/ehjci/jey156.

11. Fu W, Chen M, Ou L, Li T, Chang X, Huang R et al. Xiaoyaosan prevents atherosclerotic vulnerable plaque formation through heat shock protein/glucocorticoid receptor axis-mediated mechanism. American journal of translational research. 2019;11(9):5531-45.

12. Arnold JR, Karamitsos TD, Pegg TJ, Francis JM, Olszewski R, Searle N et al. Adenosine stress myocardial contrast echocardiography for the detection of coronary artery disease: a comparison with coronary angiography and cardiac magnetic resonance. JACC Cardiovascular imaging. 2010;3(9):934-43. doi:10.1016/j.jcmg.2010.06.011.

13. Malm S, Frigstad S, Torp H, Wiseth R, Skjarpe T. Quantitative adenosine real-time myocardial contrast echocardiography for detection of angiographically significant coronary artery disease. Journal of the American Society of Echocardiography : official publication of the American Society of Echocardiography. 2006;19(4):365-72. doi:10.1016/j.echo.2005.10.026.

14. Amzulescu MS, Langet $H$, Saloux E, Manrique A, Slimani A, Allain P, Roy C, de Meester $C$, Pasquet A, Somphone O, De Craene M, Vancraeynest D, Pouleur AC, Vanoverschelde JL, Gerber BL. Improvements of Myocardial Deformation Assessment by Three-Dimensional Speckle-Tracking versus Two-Dimensional Speckle-Tracking Revealed by Cardiac Magnetic Resonance Tagging. J Am Soc Echocardiogr. 2018 Sep;31(9):1021-1033.e1. doi: 10.1016/j.echo.2018.04.009.

15. Wang RR, Tian T, Li SQ, Leng XP, Tian JW. Assessment of Left Ventricular Global Myocardial Work in Patients With Different Degrees of Coronary Artery Stenosis by Pressure-Strain Loops Analysis. Ultrasound in medicine \& biology. 2021;47(1):33-42. doi:10.1016/j.ultrasmedbio.2020.09.017.

16. Edwards NFA, Scalia GM, Shiino K, Sabapathy S, Anderson B, Chamberlain R et al. Global Myocardial Work Is Superior to Global Longitudinal Strain to Predict Significant Coronary Artery Disease in Patients With Normal Left Ventricular Function and Wall Motion. Journal of the American Society of 
Echocardiography : official publication of the American Society of Echocardiography. 2019;32(8):947-57. doi:10.1016/j.echo.2019.02.014.

17. Prinzen FW, Vernooy K, Auricchio A. Cardiac resynchronization therapy: state-of-the-art of current applications, guidelines, ongoing trials, and areas of controversy. Circulation. 2013;128(22):2407-18. doi:10.1161/CIRCULATIONAHA.112.000112.

18. Liu X, Sun C, Tian J, Liu X, Fang S, Xi X et al. Shrinkage as a potential mechanism of recurrent clinical events in patients with a large vulnerable plaque. Journal of cardiovascular medicine. 2019;20(8):518-24. doi:10.2459/JCM.0000000000000783.

19. Lee PW, Zhang Q, Yip GW, Wu L, Lam YY, Wu EB et al. Left ventricular systolic and diastolic dyssynchrony in coronary artery disease with preserved ejection fraction. Clinical science. 2009;116(6):521-9. doi:10.1042/CS20080100.

20. Wang J, Abraham TP, Korinek J, Urheim S, McMahon EM, Belohlavek M. Delayed onset of subendocardial diastolic thinning at rest identifies hypoperfused myocardium. Circulation. 2005;111(22):2943-50. doi:10.1161/circulationaha.104.482984.

21. Duchenne J, Turco A, Unlu S, Pagourelias ED, Vunckx K, Degtiarova G et al. Left Ventricular Remodeling Results in Homogenization of Myocardial Work Distribution. Circulation Arrhythmia and electrophysiology. 2019;12(5):e007224. doi:10.1161/CIRCEP.118.007224.

22. Przewlocka-Kosmala M, Marwick TH, Mysiak A, Kosowski W, Kosmala W. Usefulness of myocardial work measurement in the assessment of left ventricular systolic reserve response to spironolactone in heart failure with preserved ejection fraction. European heart journal cardiovascular Imaging. 2019;20(10):1138-46. doi:10.1093/ehjci/jez027.

\section{Tables}

\section{Table 1 Baseline characteristics of the study population}




\begin{tabular}{|c|c|c|c|}
\hline Variable & CAD patients $(\mathrm{N}=62)$ & No CAD $(\mathrm{N}=17)$ & $\mathrm{p}$-value \\
\hline Age (years) & $63.32 \pm 10.38^{*}$ & $56.24 \pm 10.86$ & 0.016 \\
\hline Male Sex, n (\%) & $40(64.51 \%)$ & $8(47.05 \%)$ & 0.192 \\
\hline Heart rate (bpm) & $69.54 \pm 8.33$ & $71.06 \pm 8.98$ & 0.491 \\
\hline Body mass index $\left(\mathrm{kg} / \mathrm{m}^{2}\right)$ & $23.90 \pm 3.54$ & $23.03 \pm 2.77$ & 0.354 \\
\hline $\mathrm{BSA}\left(\mathrm{m}^{2}\right)$ & $1.77 \pm 0.20$ & $1.71 \pm 0.25$ & 0.336 \\
\hline $\mathrm{SBP}(\mathrm{mmHg})$ & $131.35 \pm 12.12^{\star}$ & $124.89 \pm 10.18$ & 0.048 \\
\hline $\mathrm{DBP}(\mathrm{mmHg})$ & $78.60 \pm 9.59$ & $74.71 \pm 7.64$ & 0.127 \\
\hline Hyperlipidemia, n (\%) & $6(9.67 \%)$ & $1(5.88 \%)$ & 0.614 \\
\hline Hypertension, n (\%) & $38(61.29 \%)$ & $7(41.17 \%)$ & 0.138 \\
\hline Diabetes mellitus, n (\%) & $11(17.74 \%)$ & $2(11.76 \%)$ & 0.556 \\
\hline Current smokers, n (\%) & $28(45.16 \%)^{*}$ & $3(17.64 \%)$ & 0.040 \\
\hline Gensini score & $35.27 \pm 30.26$ & & \\
\hline \multicolumn{4}{|c|}{ Conventional echocardiographic parameters } \\
\hline LVDd (mm) & $47.23 \pm 3.45$ & $47.23 \pm 3.40$ & 0.992 \\
\hline LVDs (mm) & $30.50 \pm 2.29$ & $30.35 \pm 2.34$ & 0.816 \\
\hline IVSd (mm) & $10.10 \pm 1.20$ & $9.4 \pm 1.82$ & 0.058 \\
\hline LVPWd (mm) & $9.24 \pm 1.62$ & $8.76 \pm 0.56$ & 0.237 \\
\hline Biplane LVEF (\%) & $64.63 \pm 1.17$ & $65.00 \pm 1.87$ & 0.524 \\
\hline $\mathrm{SV} / \mathrm{BSA}\left(\mathrm{ml} / \mathrm{m}^{2}\right)$ & $38.92 \pm 8.94$ & $40.32 \pm 7.19$ & 0.555 \\
\hline $\mathrm{E} / \mathrm{A}$ & $0.81 \pm 0.23$ & $0.92 \pm 0.30$ & 0.138 \\
\hline$E / e$ & $10.13 \pm 3.42$ & $9.85 \pm 3.46$ & 0.765 \\
\hline
\end{tabular}

BSA, body surface area; SBP, Systolic blood pressure; DBP, diastolic blood pressure; LVDd, LV dimension in end diastole; LVDs, LV dimension in end systole; IVSd, interventricular septal thickness in diastole; LVPWd, LV posterior wall thickness in diastole; LVEF, left ventricular ejection fraction; SV, stroke volume.

Data are presented as mean \pm SD or as number(percentage).

*Significantly different $(p<0.05)$ compared with the control (No CAD group).

Table 2 GLS and GMW parameters in CAD patients and control subjects 


\begin{tabular}{|llll|}
\hline & CAD patients $(\mathrm{N}=62)$ & No CAD $(\mathrm{N}=17)$ & p value \\
\hline GLS (\%) & $\mathbf{- 1 7 . 0 0 \pm 2 . 1 2 ^ { * }}$ & $-\mathbf{1 9 . 8 2} \pm 3.92$ & $<0.001$ \\
\hline GWE (\%) & $\mathbf{9 1 . 6 1 \pm 2 . 8 2 ^ { * }}$ & $\mathbf{9 5 . 2 9 \pm 2 . 0 5}$ & $<0.001$ \\
\hline GWI (mmHg \%) & $1678.37 \pm 281.11$ & $1816.94 \pm 285.93$ & 0.077 \\
\hline GCW (mmHg \%) & $2015.34 \pm 298.56$ & $2156.88 \pm 326.22$ & 0.094 \\
\hline GWW (mmHg \%) & $\mathbf{1 4 2 . 4 4 \pm 6 2 . 9 0 ^ { * }}$ & $\mathbf{9 0 . 5 9 \pm 5 3 . 2 8}$ & $\mathbf{0 . 0 0 3}$ \\
\hline
\end{tabular}

GLS, global longitudinal strain; GWE, global myocardial work efficiency; GWI, global myocardial work index; GCW, global constructive work; GWW, global wasted work.

Data are presented as mean \pm SD.

*Significantly different $(p<0.05)$ compared with the control (No CAD group).

Table 3 GLS and GMW parameters in three different GS groups

\begin{tabular}{|llllll|}
\hline & $\begin{array}{l}\text { No CAD } \\
(\mathrm{n}=17)\end{array}$ & $\begin{array}{l}\text { Low-GS } \\
(\mathrm{n}=21)\end{array}$ & $\begin{array}{l}\text { Mid-GS } \\
(\mathrm{n}=22)\end{array}$ & $\begin{array}{l}\text { High-GS } \\
(\mathrm{n}=19)\end{array}$ & $\begin{array}{l}\text { Correlation } \\
\text { coefficient }\end{array}$ \\
\hline with GS
\end{tabular}

GLS, global longitudinal strain; GWE, global myocardial work efficiency; GWI, global myocardial work index; GCW, global constructive work; GWW, global wasted work.

Data are presented as mean \pm SD.

*Significantly different $(p<0.05)$ compared with the control (No CAD group). 
Table 4 Receiver operating characteristic curve analysis for the detection of high-GS

\begin{tabular}{|llllll|}
\hline & GLS (\%) & GWE (\%) & GWI (mmHg\%) & GCW (mmHg\%) & GWW (mmHg\%) \\
\hline AUC(SE) & 0.677 & 0.839 & 0.669 & 0.677 & 0.796 \\
\hline AUC 95\%Cl & $0.546-0.790$ & $0.723-0.920$ & $0.538-0.783$ & $0.546-0.790$ & $0.675-0.888$ \\
\hline Cutoff value & $\leq 16.0$ & $\leq 91$ & $\leq 1541$ & $\leq 1712$ & $>142$ \\
\hline sensitivity & $61.90 \%$ & $76.19 \%$ & $57.24 \%$ & $38.10 \%$ & $76.19 \%$ \\
\hline specificity & $70.73 \%$ & $73.17 \%$ & $80.49 \%$ & $97.56 \%$ & $73.17 \%$ \\
\hline
\end{tabular}

GLS, global longitudinal strain; GWE, global myocardial work efficiency; GWI, global myocardial work index; GCW, global constructive work; GWW, global wasted work; AUC, area under the curve.

Table 5 Univariate and multivariate logistic regression analyses of variables predictive of high-GS

\begin{tabular}{|lllll|}
\hline Variables & \multicolumn{3}{l}{ Univariate analysis } & Multivariate analysis \\
\cline { 2 - 5 } & Beta & p value & Beta & p value \\
\hline $\begin{array}{l}\text { Dependent variable } \\
\text { Gensini score }\end{array}$ & & & \\
\hline Independent variables & & & \\
\hline GLS (\%) & 0.328 & 0.019 & & \\
\hline GWE (\%) & $\mathbf{3 7 . 3 2 8}$ & $\mathbf{0 . 0 0 2}$ & $\mathbf{3 9 . 3 3 0}$ & $\mathbf{0 . 0 0 1}$ \\
\hline GWI (mmHg \%) & 0.002 & 0.042 & & \\
\hline GCW (mmHg \%) & 0.002 & 0.037 & & \\
\hline GWW (mmHg \%) & -0.013 & 0.007 & \\
\hline
\end{tabular}

GLS, global longitudinal strain; GWE, global myocardial work efficiency; GWI, global myocardial work index; GCW, global constructive work; GWW, global wasted work.

\section{Figures}




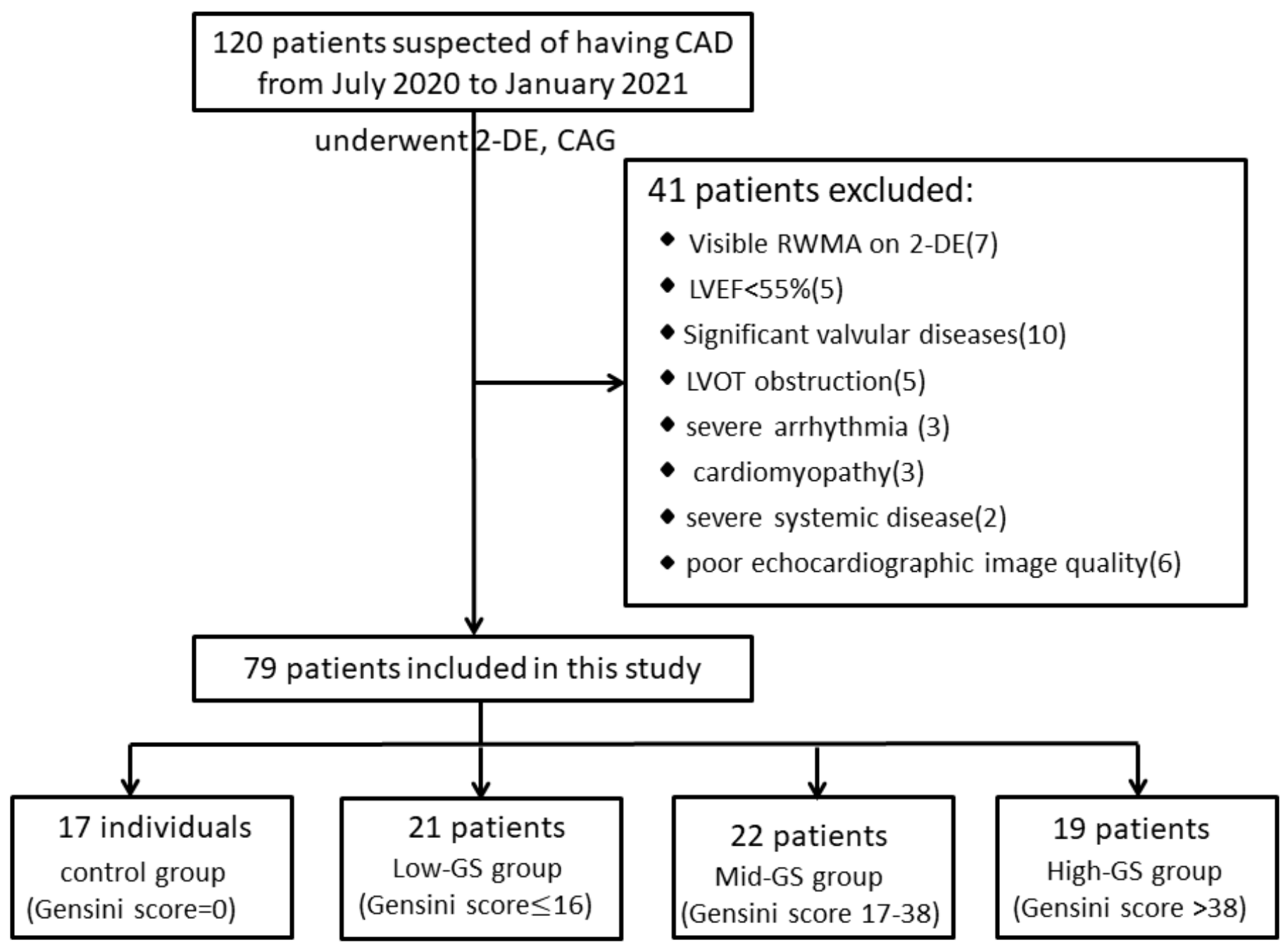

Figure 1

Inclusion of patients in the study 


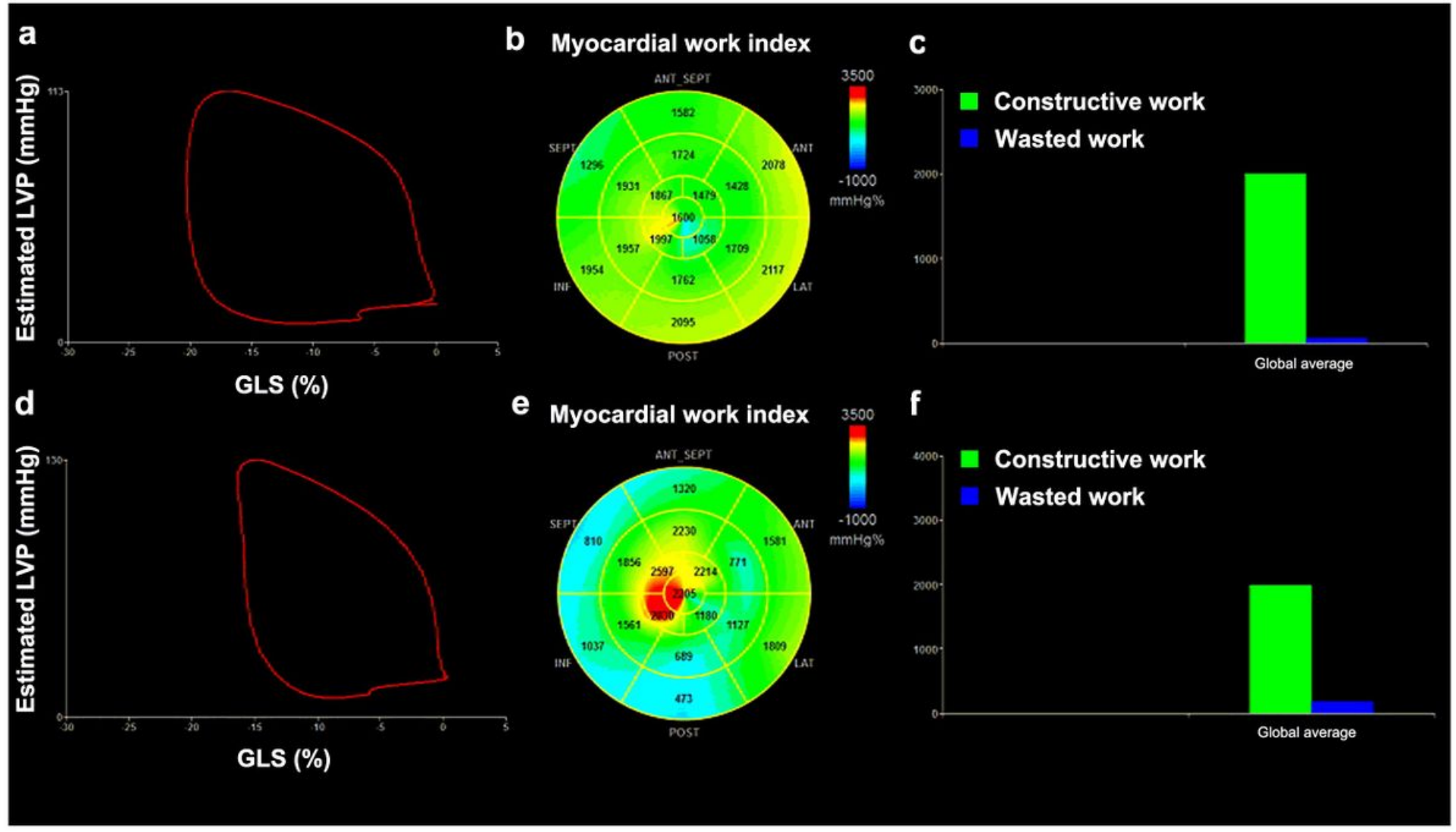

\section{Figure 2}

Representational global myocardial work parameters diagram Non-invasive left ventricular pressure-strain loop from one patient with no angiographic evidence of $C A D$ (a.) and one patient with severe $C A D(G S=42)$ (d.) respectively. The area within the loop is estimated as global MW. Visually the LV pressure-strain loop in fig. $d$ has a smaller area. Segmental bull's-eye MW plot from one patient with no angiographic evidence of CAD (b.) and one patient with severe CAD (e.) respectively. On the bull's-eye plot, there is significant reduction in fig. e demonstrated as blue-coded areas. GCW and GWW diagram from one patient with no angiographic evidence of CAD (c.) and one patient with severe CAD (f.) respectively. GS, Gensini score; MW, myocardial work; GCW, global constructive work; GWW, global wasted work. 


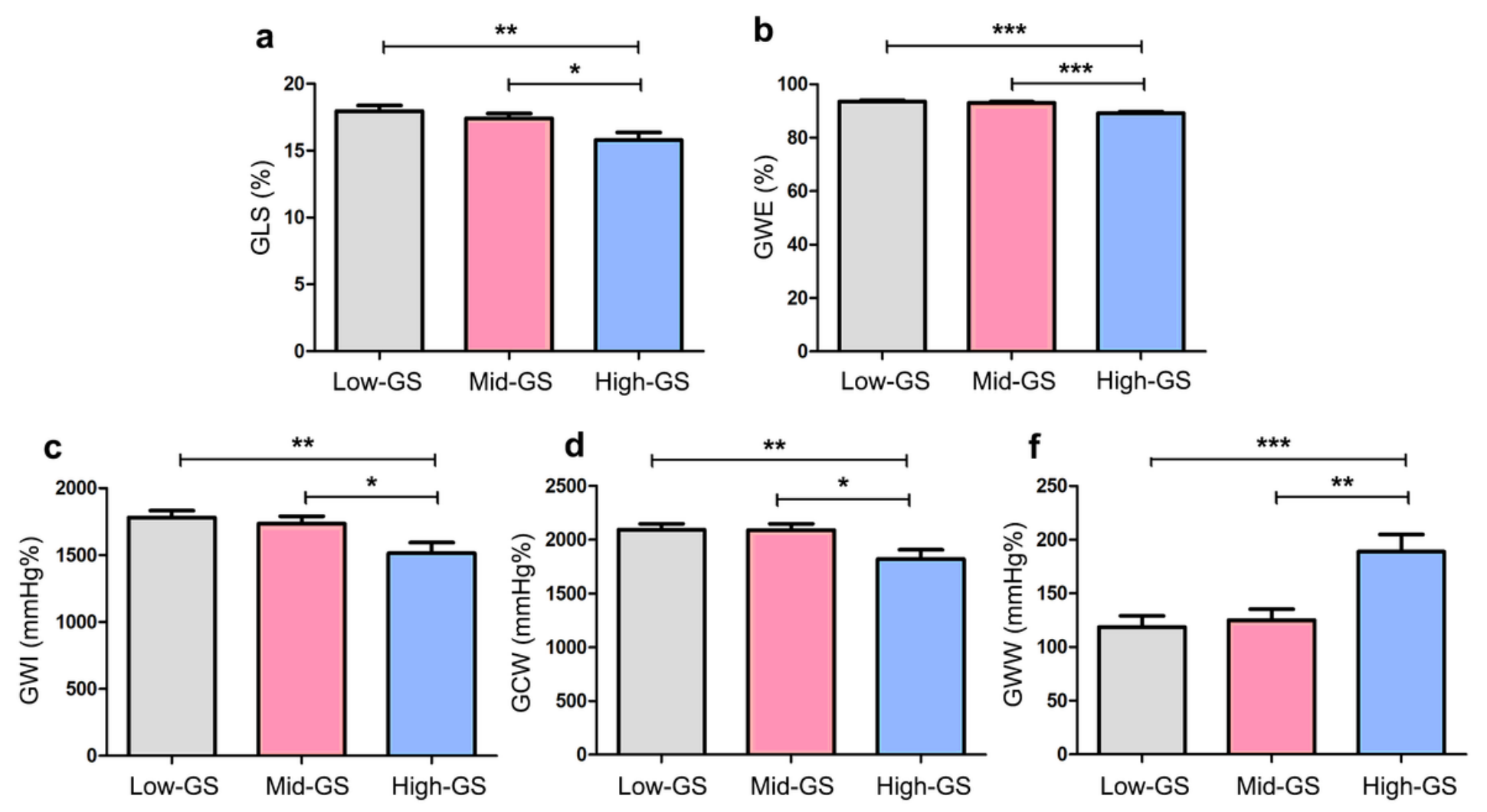

Figure 3

Comparison of GLS and GMW parameters among three GS groups. 
ROC Curves

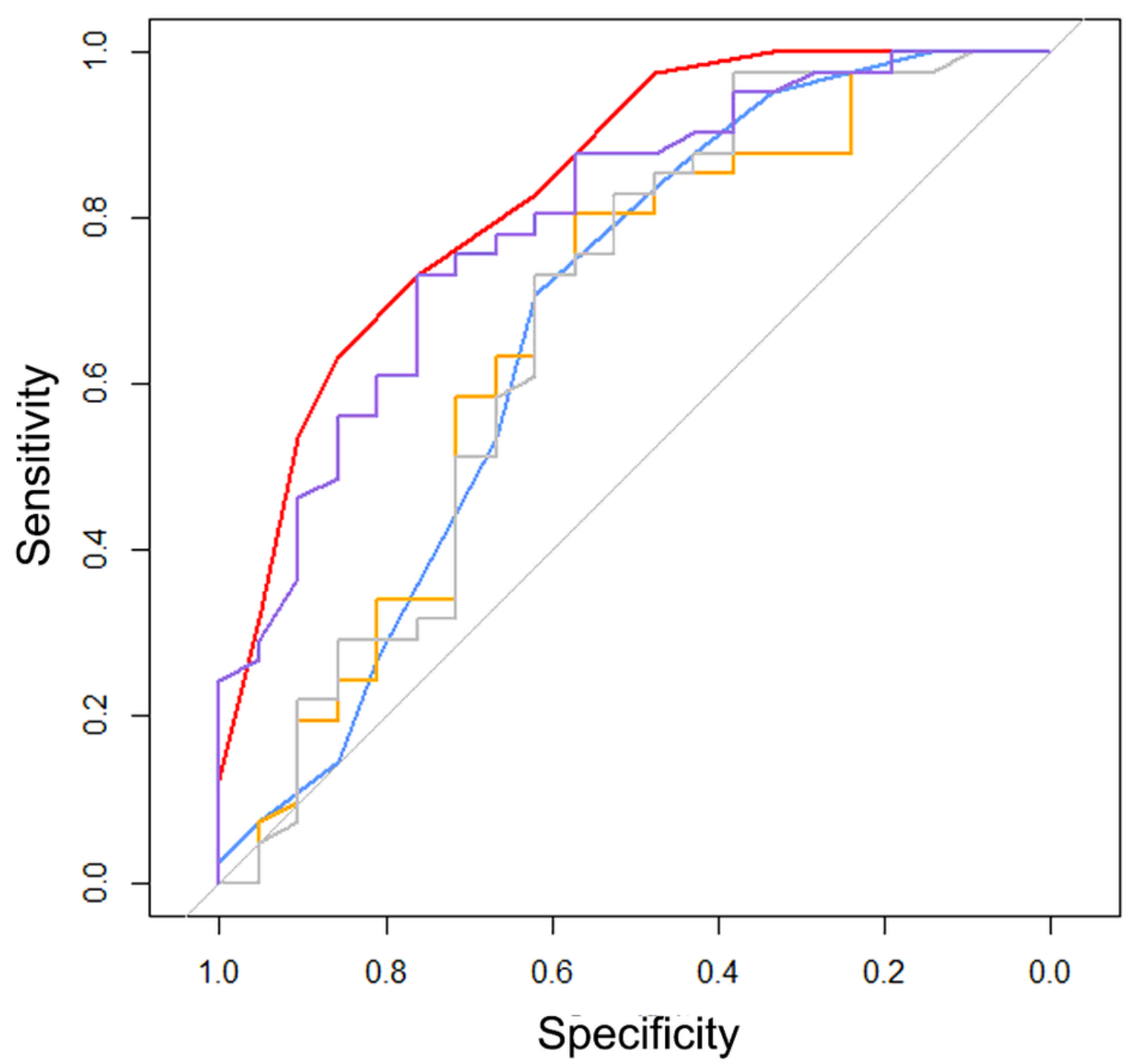

- GLS

- GWE

$-\mathrm{GWI}$

GCW

- GWW

GWW

Figure 4

ROC curves showing the diagnostic value of GLS and GMW parameters for the detection of non-RWMA CAD patients with high-GS.

\section{Supplementary Files}

This is a list of supplementary files associated with this preprint. Click to download.

- SupplementaryTable1Gensiniscoringsystem.docx

- SupplementaryTable2.docx 
- SupplementaryTable3.docx

Page 19/19 